\title{
Weak dimension-free concentration of measure
}

\author{
SERGEY G. BOBKOV ${ }^{1}$ and CHRISTIAN HOUDRÉ 2 \\ ${ }^{1}$ Department of Mathematics, Syktyvkar University, 167001 Syktyvkar, Russia. \\ E-mail: sbobkov@online.ru \\ ${ }^{2}$ Southeast Applied Analysis Center, School of Mathematics, Georgia Institute of Technology, \\ Atlanta GA 30332, USA.E-mail: houdre@math.gatech.edu
}

We study a concentration property of product probability measures with respect to the supremum distance. This property is shown to be equivalent to the conditions given by de Haan and Ridder for the stochastic boundedness of centered extreme samples.

Keywords: concentration of measure; extreme samples; isoperimetry

\section{Introduction}

Let $\mu$ be a probability measure on the real line $\mathbb{R}$, and let $\mu^{n}$ be the $n$-fold tensor product of $\mu$ with itself. Given a notion of enlargement $\operatorname{enl}(A)$ for sets $A \subset \mathbb{R}^{n}$, inequalities of isoperimetric type have the form

$$
\mu^{n}(\operatorname{enl}(A)) \geqslant R^{(n)}(\mu(A))
$$

Moreover, if $R=R^{(n)}$ is dimension-free, such inequalities are often viewed as concentration inequalities. One question of interest which will be addressed here is whether or not such a function (of course, such that $R(p)>p$ ) exists. Besides the measure, the answer depends in an essential manner on the enlargement which is usually built with the help of a metric, say $\rho$, by putting

$$
\operatorname{enl}(A)=A^{h}=\left\{x \in \mathbb{R}^{n}: \rho(x, a) \leqslant h, \text { for some } a \in A\right\},
$$

where $h>0$ is a fixed number (for $A$ compact, $A^{h}$ is the closed $h$-neighbourhood of $A$ with respect to $\rho$ ). To consider the weakest possible type of enlargement, we equip $\mathbb{R}^{n}$ with the supremum distance

$$
\rho_{\infty}(x, y)=\sup _{1 \leqslant i \leqslant n}\left|x_{i}-y_{i}\right|
$$

and consider the value

$$
R_{h}^{(n)}(p)=\inf _{\mu^{n}(A) \geqslant p} \mu^{n}\left(A^{h}\right)
$$

where the infimum is taken over all the Borel sets of measure $\mu^{n}(A) \geqslant p, p \in(0,1)$. In his 
work on isoperimetry, Talagrand (1991) made the following observation (see Proposition 5.1 there): if $\inf _{n} R_{h}^{(n)}\left(\frac{1}{2}\right)>\frac{1}{2}$, then $\mu$ has finite exponential moment, that is,

$$
\int_{\mathbb{R}} \exp (\varepsilon|x|) \mathrm{d} \mu(x)<+\infty,
$$

for some $\varepsilon>0$. In proving this result, he studied the behaviour of $\mu^{n}\left(A^{h}\right)$ for the cubes $A$. It turns out that studying the enlargements of the cubes also allows us to find necessary and sufficient conditions for the validity of the concentration inequality $\mu^{n}\left(A^{h}\right) \geqslant R(\mu(A)$ ), for some $R$ such that $R(p)>p$. This property turns out to be equivalent to the stochastic boundedness of centered extreme samples. This boundedness was previously studied by de Haan and Ridder (1979) who explicitly described the corresponding class of underlying probability measures $\mu$. For the exponential measure (and for all Lipschitz images of the exponential measure), Talagrand (1995) proved a concentration inequality for a notion of enlargement much smaller than the one defined by the supremum distance (cf. also Bobkov and Ledoux 1997). As we will see, beyond the class of Lipschitz images of the exponential measure, there exist probability distributions still enjoying some concentration property (as defined above).

Definition. A function $U$ defined on some interval $\Delta \subset \mathbb{R}$ is said to have finite modulus of continuity if, for all (equivalently, for some) $h>0$,

$$
U^{*}(h)=\sup \{|U(x)-U(y)|: x, y \in \Delta,|x-y| \leqslant h\}<+\infty .
$$

The function $U^{*}$ is then called the modulus of continuity generated by $U$. Clearly, since $U^{*}\left(h_{1}+h_{2}\right) \leqslant U^{*}\left(h_{1}\right)+U^{*}\left(h_{2}\right)$, for all $h_{1}, h_{2} \geqslant 0, U$ has finite modulus of continuity if and only if, for some $a, b \geqslant 0,|U(x)-U(y)| \leqslant a+b|x-y|$ whenever $x, y \in \Delta$.

Now let $U_{\mu}$ be defined as follows:

$$
U_{\mu}(x)=F_{\mu}^{-1}\left(\frac{1}{1+\mathrm{e}^{-x}}\right), \quad x \in \mathbb{R},
$$

where $F_{\mu}(x)=\mu((-\infty, x])$ is the distribution function of the measure $\mu$, and where

$$
F_{\mu}^{-1}(p)=\inf \left\{x \in \mathbb{R}: F_{\mu}(x) \geqslant p\right\}, \quad p \in(0,1),
$$

is the minimal quantile of order $p$ of $\mu$. The meaning of this definition is that the map $U_{\mu}$ transforms the logistic probability measure $v\left(v((-\infty, x])=\left(1+\mathrm{e}^{-x}\right)^{-1}\right)$ into the measure $\mu$. The aim of these notes is to prove the following.

Theorem 1.1. Let $p \in(0,1)$. The following properties are equivalent:

(a) There exists $h>0$ such that $\inf _{n} R_{h}^{(n)}(p)>p$.

(b) There exist $\delta>0$ and $c>0$ such that, for all $x \in \mathbb{R}$,

$$
F_{\mu}(x)-F_{\mu}(x-\delta) \geqslant c F_{\mu}(x)\left(1-F_{\mu}(x)\right) .
$$

(c) The function $U_{\mu}$ has finite modulus of continuity. In this case, for every $h>0$, setting $h^{*}=U_{\mu}^{*}(h)$, we have 


$$
\inf _{n} R_{h^{*}}^{(n)}(p) \geqslant \frac{p}{p+(1-p) \exp (-h)},
$$

with equality for $\mu=v$. In particular, the following alternative holds: either $\inf _{n} R_{h}^{(n)}(p)=p$, for all $h>0$, or $\inf _{n} R_{h}^{(n)}(p) \rightarrow 1$, as $h \rightarrow+\infty$.

In more probabilistic language, inequality (1.3) can be expressed as follows. Let $\xi_{n}$, $n \geqslant 1$, be a sequence of independent random variables defined on some probability space $(\Omega, \mathscr{F}, P)$, with common law $\mu$ and associated distribution function $F_{\mu}$. Let $\zeta$ be a logistic random variable (with law $v$ ). Then, the right-hand side of (1.3) is simply $P\left\{\zeta-m_{p}(\xi) \leqslant h\right\}$, where $m_{p}(\xi)=F_{v}^{-1}(p)$ is the quantile of order $p$ of $\zeta$. Let $f: \mathbb{R}^{n} \rightarrow \mathbb{R}$ be an arbitrary Lipschitz function, with Lipschitz constant at most 1 with respect to $\rho_{\infty}$, and let $\eta=f\left(\xi_{1}, \ldots, \xi_{n}\right)$. If (1.3) is applied to sets of the form $\{f \leqslant$ const. $\}$, it is easily seen that

$$
P\left\{\eta-m_{p}(\eta) \leqslant h^{*}\right\} \geqslant P\left\{\zeta-m_{p}(\zeta) \leqslant h\right\},
$$

for all $p \in(0,1)$ and $h>0$. Furthermore, this can be shown to be equivalent to the following property: there exists a non-decreasing function $U_{f}: \mathbb{R} \rightarrow \mathbb{R}$ with $U_{f}^{*} \leqslant U_{\mu}^{*}$ such that the random variables $\eta$ and $U_{f}(\xi)$ are identically distributed. Thus, at the level of distributions, all the random variables $f\left(\xi_{1}, \ldots, \xi_{n}\right)$ where $f$ is $\rho_{\infty}$-Lipschitz can be viewed as random variables of the form $U(\xi)$ with $U^{*} \leqslant U_{\mu}^{*}$. A simple consequence of this property is the fact that the variance $\operatorname{var}\left(f\left(\xi_{1}, \ldots, \xi_{n}\right)\right)$ can be bounded by a quantity which only depends on $U_{\mu}^{*}$. This is in particular true for the functions $f_{n}(x)=\max \left\{x_{1}, \ldots, x_{n}\right\}$ and $g_{n}(x)=$ $\min \left\{x_{1}, \ldots, x_{n}\right\}$ which play a crucial role below.

Corollary 1.2. Let $p \in(0,1)$. There exists $h>0$ such that $\inf _{n} R_{h}^{(n)}(p)>p$, if and only if the random variables $\xi_{n}$ have finite second moment and the following two conditions hold:

(1a) $\sup _{n} \operatorname{var} \max \left\{\xi_{1}, \ldots, \xi_{n}\right\}<+\infty$,

(2a) $\sup _{n} \operatorname{var} \min \left\{\xi_{1}, \ldots, \xi_{n}\right\}<+\infty$;

or equivalently, and more generally, if and only if, for any fixed $\alpha \geqslant 1$,

(1b) $\sup _{n} \mathrm{E}\left|\max \left\{\xi_{1}, \ldots, \xi_{n}\right\}-\mathrm{E} \max \left\{\xi_{1}, \ldots, \xi_{n}\right\}\right|^{\alpha}<+\infty$;

(2b) $\sup _{n} \mathrm{E}\left|\min \left\{\xi_{1}, \ldots, \xi_{n}\right\}-\mathrm{E} \min \left\{\xi_{1}, \ldots, \xi_{n}\right\}\right|^{\alpha}<+\infty$.

Moreover, in (1b) and (2b) the second expectations can be replaced by the quantiles of order $p \in(0,1)$.

The properties (1a), (2a) can also be written (together but not separately) in a weaker form as follows: for some real numbers $a_{n}$ and $b_{n}$, the random variables

$$
\max \left\{\xi_{1}, \ldots, \xi_{n}\right\}-a_{n}, \quad \min \left\{\xi_{1}, \ldots, \xi_{n}\right\}-b_{n},
$$

are stochastically bounded, or using different terminology, their distributions form a precompact family in the space of all probability measures on $\mathbb{R}$ with respect to the topology of weak convergence. That is, 
(1c) $\sup _{n} P\left\{\left|\max \left\{\zeta_{1}, \ldots, \xi_{n}\right\}-a_{n}\right|>h\right\} \rightarrow 0$, as $h \rightarrow+\infty$;

(2c) $\sup _{n} P\left\{\left|\min \left\{\xi_{1}, \ldots, \xi_{n}\right\}-b_{n}\right|>h\right\} \rightarrow 0$, as $h \rightarrow+\infty$.

De Haan and Ridder (1979) found, directly in terms of $F_{\mu}$, several necessary and sufficient conditions for (1c) (cf. their Proposition 2.2.1 and Theorem 3.1), one of which is the following property: there exist $x_{0}$ and $h_{0}$ such that, for all $x \geqslant x_{0}$ and $h \geqslant h_{0}$,

$$
1-F_{\mu}(x+h) \leqslant c\left(1-F_{\mu}(x)\right), \quad \text { for some } c \in(0,1) .
$$

When combining the above inequality with a similar inequality for $-\xi_{1}$, we arrive exactly at (1.2), with possibly another constant depending on $\mu$ only.

The description (1.2) is explicit and certainly convenient to use in the case of specific examples of probability distributions $\mu$. However, for our purposes it will be essential to connect (1.2) with the moduli of continuity. When $\mu=v$, inequality (1.3) is known (cf. Bobkov 1996, Bobkov and Houdré 1997), and in fact, it is easy to prove (1.3) transporting $v$ into $\mu$ via $U_{\mu}$. The non-trivial part of Theorem 1.1 will be to show that $U_{\mu}$ has finite modulus of continuity provided that $\inf _{n} R_{h}^{(n)}(p)>p$. Property (c) in Theorem 1.1 also allows us to make the following observation: it is possible for the tails $h \rightarrow \mu\{x \in \mathbb{R}:|x|>h\}$ to tend to zero exponentially fast (as $h \rightarrow+\infty$ ), or as fast as we want, without the products $\mu^{n}$ satisfying the concentration property (a). Indeed, given a decreasing, continuous function $\varepsilon:[0,+\infty) \rightarrow\left(0, \frac{1}{2}\right]$ with $\varepsilon(0)=\frac{1}{2}$ and $\varepsilon(h) \rightarrow 0$, as $h \rightarrow+\infty$, one can construct an even, continuous, strictly increasing function $U$ on $\mathbb{R}$ with $U(0)=0$ such that $U^{*} \equiv+\infty$ but such that the measure $\mu=v U^{-1}$, the image of $v$ under $U$, has the tails bounded by the function $\varepsilon(h)$. Thus, concentration property (a) is not determined by the tail behaviour of $\mu$.

It is of course natural to ask if there exist necessary and sufficient conditions on $F_{\mu}$ for the (stronger) concentration property $\inf _{n} R_{h}^{(n)}(p)>p$ when one takes in (1.1) the enlargement $A^{h}$ with respect to the usual Euclidean metric $\rho_{2}$ in $\mathbb{R}^{n}$. As far as we know, this question is still open. However, if we restrict ourselves in (1.1) to the class of sets $A \subset \mathbb{R}^{n}$ which are convex or whose complement is convex, the property $\inf _{n} R_{h}^{(n)}(p)>p$ will again be equivalent to (1.2) and thus we will obtain the same class of generating distributions $\mu$ (cf. Bobkov and Götze 1999). Moreover, for such and only such measures, the variances of all convex $\rho_{2}$-Lipschitz functions are bounded by a constant independent of the dimension.

The proofs of Theorem 1.1 and Corollary 1.2 are respectively given in Sections 3 and 4 . Here we also discuss property (1b) in Corollary 1.2. We start (Section 2) with characterizations of the concentration property for the distributions of maxima. The paper finishes with some remarks.

\section{Concentration of maxima}

Below and throughout, let $M_{n}=\max \left\{\xi_{1}, \ldots, \xi_{n}\right\}$.

Lemma 2.1. The following are equivalent: 
(1a) For some $p \in(0,1)$ and $h>0$, $\inf _{n} P\left\{M_{n}-m_{p}\left(M_{n}\right) \leqslant h\right\}>p$.

(1b) For some $p \in(0,1)$ and $h<0, \sup _{n} P\left\{M_{n}-m_{p}\left(M_{n}\right) \leqslant h\right\}<p$.

(1c) For a sequence of real numbers $a_{n}$, $\sup _{n} P\left\{\left|M_{n}-a_{n}\right|>h\right\} \rightarrow 0$, as $h \rightarrow+\infty$.

(1d) There exists $\varepsilon>0$ such that, for any $p \in(0,1)$,

$$
\sup _{n} \operatorname{E} \exp \left\{\varepsilon\left(M_{n}-m_{p}\left(M_{n}\right)\right)\right\}<+\infty .
$$

(2) For all (equivalently, for some) $a \in \mathbb{R}$, the function $U(x)=F_{\mu}^{-1}(1 /(1+\exp (-x)))$ has finite modulus of continuity in the interval $x \geqslant a$.

The equivalence between (1c) and (1d) is essentially known and is due to de Haan and Ridder (1979).

Proof. It will be convenient to work with another (equivalent) condition:

$\left(2^{\prime}\right)$ For all (equivalently, for some) $a \in \mathbb{R}$, the function $V(x)=F_{\mu}^{-1}(\exp (-\exp (-x)))$, $x \geqslant a$, has finite modulus of continuity. In addition, for any $p \in(0,1)$ and $h>0$,

$$
\sup _{n} P\left\{M_{n}-m_{p}\left(M_{n}\right)>V_{p}^{*}(h)\right\} \leqslant P\left\{Z-m_{p}(Z)>h\right\},
$$

where the random variable $Z$ has distribution $P\{Z \leqslant x\}=\exp (-\exp (-x))$, and where $V_{p}^{*}$ is a modulus of continuity generated by $V$ on the interval $[-\log \log (1 / p),+\infty)$.

The main step in the proof is the implication $(1 \mathrm{a}) \Rightarrow\left(2^{\prime}\right)$. Let $p, q \in(0,1)$ and $h_{0}>0$ be such that

$$
\inf _{n} P\left\{M_{n}-m_{p}\left(M_{n}\right) \leqslant h_{0}\right\} \geqslant q>p,
$$

that is, such that $F_{\mu}\left(F_{\mu}^{-1}\left(p^{1 / n}\right)+h_{0}\right) \geqslant q^{1 / n}$. By the very definition of $F_{\mu}^{-1}$, this implies

$$
F_{\mu}^{-1}\left(q^{1 / n}\right)-F_{\mu}^{-1}\left(p^{1 / n}\right) \leqslant h_{0} .
$$

Putting $a_{0}=-\log \log (1 / p), b_{0}=-\log \log (1 / q)$, (2.2) can be rewritten as

$$
V\left(b_{0}+\log (n)\right)-V\left(a_{0}+\log (n)\right) \leqslant h_{0},
$$

which holds for all $n \geqslant 1$. We need to deduce from (2.3) that

$$
V_{p}^{*}(h)=\sup \{V(y)-V(x): a \leqslant x \leqslant y, y-x \leqslant h\}<+\infty,
$$

whenever $a \in \mathbb{R}$ and $h>0$. Now, fix any real number $c$ such that $1<c<\exp \left(b_{0}-a_{0}\right)$, and let $n_{0}$ be any positive integer such that

$$
\log \left(n_{0}+1\right)-\log \left(n_{0}\right) \leqslant b_{0}-a_{0}, \quad\left(\exp \left(b_{0}-a_{0}\right)-c\right) n_{0} \geqslant 1 .
$$

Clearly, $V$ is a non-decreasing function on $\mathbb{R}$, hence the property (2.4) does not depend on $a$ and $h$, so we can let $a=a_{0}+\log \left(n_{0}\right), h=h_{0}$. Thus, in order to prove (2.4), it can be assumed that $a_{0}+\log \left(n_{0}\right) \leqslant x \leqslant y \leqslant x+h_{0}$.

Now define a sequence $n_{k}, k \geqslant 1$, recursively in the following way: let $n_{1}$ be the largest integer such that $a_{0}+\log \left(n_{1}\right) \leqslant x$; and if $k \geqslant 1$, let $n_{k+1}$ be the largest integer such that $a_{0}+\log \left(n_{k+1}\right) \leqslant b_{0}+\log \left(n_{k}\right)$. Then $n_{0} \leqslant n_{k}<n_{k+1}$, for all $k \geqslant 1$, since $a_{0}+\log \left(n_{k}+\right.$ $1) \leqslant b_{0}+\log \left(n_{k}\right)$ which holds due to (2.5) and since $n_{k} \geqslant n_{0}$.

Denote by $K$ the smallest $k$ such that $b_{0}+\log \left(n_{k}\right) \geqslant y$. By construction, the intervals 
$\Delta_{k}=\left[a_{0}+\log \left(n_{k}\right), b_{0}+\log \left(n_{k}\right)\right], 1 \leqslant k \leqslant K$, cover the interval $[x, y]$. Therefore, using (2.3), we obtain

$$
V(y)-V(x) \leqslant \sum_{k=1}^{K} V\left(b_{0}+\log \left(n_{k}\right)\right)-V\left(a_{0}+\log \left(n_{k}\right)\right) \leqslant K h_{0} .
$$

Our aim is now to find an estimate of $K$ depending on $y-x$; we would then have an estimate for $V(y)-V(x)$ in terms of $y-x$. Denote by $[u]$ the integer part of a real $u$. Then $n_{k+1}=\left[\exp \left(b_{0}-a_{0}\right) n_{k}\right]$, hence

$$
n_{k+1} \geqslant \exp \left(b_{0}-a_{0}\right) n_{k}-1 \geqslant c n_{k},
$$

since $n_{k} \geqslant n_{0}$ and since $\exp \left(b_{0}-a_{0}\right) n_{0} \geqslant c n_{0}+1$. By induction, it is easy to see that $n_{k} \geqslant c^{k-1} n_{1}$, that is, $\log \left(n_{k}\right) \geqslant(k-1) \log (c)+\log \left(n_{1}\right)$. Thus, the inequality $b_{0}+$ $\log \left(n_{k}\right) \geqslant y$ follows from $b_{0}+(k-1) \log (c)+\log \left(n_{1}\right) \geqslant y$. The last inequality can be rewritten as

$$
k \geqslant 1+\frac{y-b_{0}-\log \left(n_{1}\right)}{\log (c)} .
$$

By the very definition of $n_{1}$, we also have $\log \left(n_{1}+1\right)>x-a_{0}$, and since $\log \left(n_{1}+1\right)-$ $\log \left(n_{1}\right) \leqslant b_{0}-a_{0}$, we have the estimate $\log \left(n_{1}\right)>x-b_{0}$. Therefore, (2.6) is fulfilled if we take $k$ such that $k \geqslant 1+(y-x) / \log (c)$. Hence

$$
K \leqslant 2+\frac{y-x}{\log (c)}
$$

We thus have proved (2.4) and the first part of $\left(2^{\prime}\right)$.

To prove the second part of $\left(2^{\prime}\right)$, fix $p \in(0,1), h>0$, and set $r=P\left\{Z-m_{p}(Z) \leqslant h\right\}$, $a=-\log \log (1 / p), b=-\log \log (1 / r)$. Then, as easily verified, $b-a=h$. As previously seen, inequalities of the form

$$
P\left\{M_{n}-m_{p}\left(M_{n}\right) \leqslant V_{p}^{*}(h)\right\} \geqslant r
$$

are equivalent to

$$
V(b+\log (n))-V(a+\log (n)) \leqslant V_{p}^{*}(h)
$$

(this is (2.3) with $V_{p}^{*}(h)$ instead of $h_{0}$ ). Since $b-a=h$, the above inequality holds true by the very definition of $V_{p}^{*}$. It just remains to note that (2.7) and (2.1) coincide.

$\left(2^{\prime}\right) \Rightarrow(1 \mathrm{a})$. Let $p=\exp (-\exp (-a))$. Then, as shown in the previous steps, (1a) holds if and only if (2.4) holds for some $b>a$, some $h>0$ and all $n \geqslant 1$. But (2.4) holds for all $b>a$ and $h>0$ since $V(b+\log (n))-V(a+\log (n)) \leqslant V_{p}^{*}(b-a)$.

$(2) \Rightarrow\left(2^{\prime}\right)$. Note that $U(x)=V(T(x))$, where $T(x)=-\log \log \left(1+\mathrm{e}^{-x}\right)$. Then $T$ is an increasing bijection from $\mathbb{R}$ to $\mathbb{R}$, and has a finite Lipschitz constant on every interval $[a,+\infty)$, and similarly for its inverse $T^{-1}$. Therefore, $U$ has finite modulus of continuity on $[a,+\infty)$ if and only if $V$ has finite modulus of continuity on $[a,+\infty)$.

(1a) $\Rightarrow(1 \mathrm{~b})$. Simply note (recalling (2.2)) that, for all $0<p<q<1$ and all $h>0$,

$$
P\left\{M_{n}-m_{p}\left(M_{n}\right)<h\right\} \geqslant q \Rightarrow P\left\{M_{n}-m_{q}\left(M_{n}\right) \leqslant-h\right\}<p .
$$


$\left(2^{\prime}\right) \Rightarrow(1 \mathrm{c})$. Let $a_{n}=m_{p}\left(M_{n}\right)$. Then (2.1) implies that $\sup _{n} P\left\{M_{n}-a_{n}>h\right\} \rightarrow 0$, as $h \rightarrow 0$, so we need to estimate the left deviations $\sup _{n} P\left\{M_{n}-a_{n}<h\right\}$. Take $a_{n}=m_{q}\left(M_{n}\right)$ with fixed (but arbitrary) $q \in(0,1)$. Inserting in (2.8) $V_{p}^{*}(h)+\varepsilon$ instead of $h(\varepsilon>0)$, and letting $\varepsilon \rightarrow 0^{+}$, gives, for all $p \in(0, q)$ and for all $h>0$ :

$$
\begin{gathered}
P\left\{M_{n}-m_{p}\left(M_{n}\right) \leqslant V_{p}^{*}(h)\right\} \geqslant q \Leftrightarrow \\
P\left\{M_{n}-m_{q}\left(M_{n}\right) \leqslant-h^{\prime}\right\}<p, \quad \text { for all } h^{\prime}>V_{p}^{*}(h) .
\end{gathered}
$$

If $p$ is chosen so that $q=P\left\{Z-m_{p}(Z) \leqslant h\right\}$, that is, $\log \log (1 / p)-\log \log (1 / q)=h$, then (2.9) is true, thanks to (2.4), hence (2.10) holds. It remains to note that $p \rightarrow 0$ as $h \rightarrow+\infty$, and since $V_{p}^{*}(h)$ is finite, we conclude that $P\left\{M_{n}-a_{n} \leqslant-h^{\prime}\right\} \rightarrow 0$ as $h^{\prime} \rightarrow+\infty$.

(1c) $\Rightarrow$ (1a). Without loss of generality, we may prove (1a) for $p=\frac{1}{2}$. By assumption, there exists $h_{0}$ such that $P\left\{\left|M_{n}-a_{n}\right|>h_{0}\right\}<\frac{1}{2}$, for all $n \geqslant 1$. Hence, $\left|m_{p}\left(M_{n}\right)-a_{n}\right| \leqslant h_{0}$. Therefore,

$$
\sup _{n} P\left\{M_{n}-m_{p}\left(M_{n}\right)>h\right\} \leqslant \sup _{n} P\left\{M_{n}-a_{n}>h-h_{0}\right\} \rightarrow 0, \quad \text { as } h \rightarrow+\infty .
$$

Finally, it is clear that $\left(2^{\prime}\right)$ implies (1d) which in turn implies (1a). Thus, Lemma 2.1 is proved.

\section{Proof of Theorem 1.1}

(a) $\Rightarrow$ (c). Assume that there exist $h>0$ and $0<p<q<1$, such that $\inf _{n} R_{h}^{(n)}(p) \geqslant q$. Thus, for all integers $n \geqslant 1$ and for all Borel sets $A \subset \mathbb{R}^{n}$ with $\mu^{n}(A) \geqslant p$, we have

$$
\mu^{n}\left(A^{h}\right) \geqslant q \text {. }
$$

Applying (3.1) first to the cubes $A_{n}(p)=\left(-\infty, F_{u}^{-1}\left(p^{1 / n}\right)\right]^{n}$, and since $\mu^{n}\left(A_{n}(p)\right)=$ $F_{\mu}^{n}\left(F_{\mu}^{-1}\left(p^{1 / n}\right)\right) \geqslant p$ and $A_{n}(p)^{h}=\left(-\infty, F_{\mu}^{-1}\left(p^{1 / n}\right)+h\right]^{n}$, gives

$$
P\left\{M_{n}-m_{p}\left(M_{n}\right) \leqslant h\right\}=F_{\mu}^{n}\left(F_{\mu}^{-1}\left(p^{1 / n}\right)+h\right)=\mu^{n}\left(A_{n}(p)^{h}\right) \geqslant q,
$$

that is, property (1a) of Lemma 2.1 is fulfilled. Therefore, so is property (2): the function $U_{\mu}$ has finite modulus of continuity on the interval $[0,+\infty)$. Now, apply (3.1) to the cubes $B_{n}(p)=\left[-F_{\mu}^{-1}\left(1-p^{1 / n}\right),+\infty\right)^{n}$. By applying the same argument to the random variables $-\xi_{n}, n \geqslant 1$, we see that $U_{\mu}$ has finite modulus of continuity on $(-\infty, 0]$, and therefore on the whole real line.

(c) $\Rightarrow($ a). It is known that, for the measure $v$ with $v((-\infty, x])=1 /(1+\exp (-x))$,

$$
v^{n}\left(A^{h}\right) \geqslant \frac{p}{p+(1-p) \exp (-\mathrm{h})},
$$

whenever $v^{n}(A) \geqslant p$, with equality at the standard half-spaces $A=\left\{x: x_{1} \leqslant\right.$ const. $\}-$ different proofs of (3.2) can also be found in Bobkov (1996) and in Bobkov and Houdré (1997, Corollary 15.3). Introduce the function $i\left(x_{1}, \ldots, x_{n}\right)=\left(U_{\mu}\left(x_{1}\right), \ldots, U_{\mu}\left(x_{n}\right)\right)$ which transforms $v^{n}$ into $\mu^{n}$. Let $h>0, h^{*}=U_{\mu}^{*}(h)$. Now observe the following inclusion: for any set $A \subset \mathbb{R}^{n}$, 


$$
\left(i^{-1}(A)\right)^{h} \subset i^{-1}\left(A^{h^{*}}\right)
$$

Indeed, if $x \in\left(i^{-1}(A)\right)^{h}$, then for some $y \in i^{-1}(A)$ we have $\rho_{\infty}(x, y) \leqslant h$, that is, $\left|x_{k}-y_{k}\right| \leqslant h$, for all $1 \leqslant k \leqslant n$. Since $i(y)=\left(U_{\mu}\left(y_{1}\right), \ldots, U_{\mu}\left(y_{n}\right)\right) A$ and since $\left|U_{\mu}\left(x_{k}\right)-U_{\mu}\left(y_{k}\right)\right| \leqslant h^{*}$, we obtain $\rho_{\infty}(i(x), i(y)) \leqslant h$, and therefore $i(x) \in A^{h^{*}}$, hence $x \in i^{-1}\left(A^{h^{*}}\right)$. Now combine (3.2) and (3.3) to prove (1.3). Let $v^{n}(A)=\mu^{n}\left(i^{-1}(A)\right) \geqslant p$; then

$$
\mu^{n}\left(A^{h^{*}}\right)=v^{n}\left(i^{-1}\left(A^{h^{*}}\right)\right) \geqslant v^{n}\left(\left(i^{-1}(A)\right)^{h}\right) \geqslant \frac{p}{p+(1-p) \exp (-h)} .
$$

To complete the proof of Theorem 1.1, it remains to see the equivalence of (b) and (c). A quantitative version of this equivalence is given by the following lemma.

Lemma 3.1. Given $\delta>0$ and $c>0$, assume that

$$
F_{\mu}(x)-F_{\mu}(x-\delta) \geqslant c F_{\mu}(x)\left(1-F_{\mu}(x), \quad \text { for all } x \in \mathbb{R} .\right.
$$

Then $U_{\mu}^{*}(h) \leqslant \delta$ with $h=\log (1+c)$. Conversely, if, for some positive $h$ and $\delta, U_{\mu}^{*}(h) \leqslant \delta$, then (3.4) holds with $c=1-\mathrm{e}^{-h}$.

Proof. Recall that $U_{\mu}(x)=F_{\mu}^{-1}\left(F_{\nu}(x)\right), x \in \mathbb{R}$. Define the values $U_{\mu}(-\infty)$ and $U_{\mu}(+\infty)$ in the usual limiting sense. Define also the function $U_{\mu}^{-1}: \mathbb{R} \rightarrow[-\infty,+\infty]$ by $U_{\mu}^{-1}(a)=$ $F_{\nu}^{-1}\left(F_{\mu}(a)\right)$ so that $F_{\mu}(a)=F_{\nu}\left(U_{\mu}^{-1}(a)\right)$, for all $a \in \mathbb{R}$. It is straightforward to verify that, for all $z \in \mathbb{R}$,

$$
U_{\mu}\left(U_{\mu}^{-1}(z)\right) \leqslant z \leqslant U_{\mu}^{-1}\left(U_{\mu}(z)\right)
$$

To prove the lemma, we first assume that $U_{\mu}^{*}(h) \leqslant \delta$ and derive (3.4). Fix $x \in \mathbb{R}$ and assume that $0<F(x-\delta) \leqslant F(x)<1$ (otherwise (3.4) is immediate). Thus, the value $a=U_{\mu}^{-1}(x-\delta)$ is finite. By the assumption and by the left inequality in (3.5) with $z=x-\delta$, we obtain

$$
U_{\mu}(a+h)-\delta \leqslant U_{\mu}(a) \leqslant x-\delta,
$$

that is, $x \geqslant U_{\mu}(a+h)$. Taking $U_{\mu}^{-1}$ of both sides and applying the right-hand inequality in (3.5), we obtain

$$
U_{\mu}^{-1}(x) \geqslant a+h=U_{\mu}^{-1}(x-\delta)+h .
$$

We also need the following trivial inequalities for the logistic distribution:

$$
1-\mathrm{e}^{v-u} \leqslant \frac{F_{\nu}(u)-F_{\nu}(v)}{F_{\nu}(u)\left(1-F_{\nu}(u)\right)} \leqslant \mathrm{e}^{u-v}-1, \quad \text { for all } u \geqslant v .
$$

Applying (3.6) and the left-hand inequality in (3.7) with $u=U_{\mu}^{-1}(x), v=U_{\mu}^{-1}(x)-h$, we obtain that 


$$
\begin{aligned}
F_{\mu}(x)-F_{\mu}(x-\delta) & =F_{\nu}\left(U_{\mu}^{-1}(x)\right)-F_{\nu}\left(U_{\mu}^{-1}(x-\delta)\right) \\
& \geqslant F_{\nu}\left(U_{\mu}^{-1}(x)\right)-F_{\nu}\left(U_{\mu}^{-1}(x)-h\right) \\
& \geqslant\left(1-\mathrm{e}^{-h}\right) F_{\nu}\left(U_{\mu}^{-1}(x)\right)\left(1-F_{\nu}\left(U_{\mu}^{-1}(x)\right)\right) \\
& =\left(1-\mathrm{e}^{-h}\right) F_{\mu}(x)\left(1-F_{\mu}(x)\right) .
\end{aligned}
$$

This proves the second assertion of Lemma 3.1. The first assertion can be proved in a similar way, with the help of the right-hand inequality in (3.7). Actually it is also proved, as Lemma 4.5 in Bobkov and Götze (1999), for the related two-sided exponential distribution (instead of $v)$, with $h=\log (1+c / 2)$.

Corollary 3.2. In Theorem 1.1, for every $h>0$, the concentration inequality (1.3) holds with

$$
h^{*}=\left(\frac{h}{\log (1+c)}+1\right) \delta,
$$

where $c$ and $\delta$ are from (1.2).

Indeed, by the first assertion of Lemma 3.1, $U_{\mu}^{*}(\log (1+c)) \leqslant \delta$. Hence, for any integer $k \geqslant 1$, we have $U_{\mu}^{*}(k \log (1+c)) \leqslant k \delta$. Taking $k=[h / \log (1+c)]+1$, we obtain $h \leqslant$ $k \log (1+c)$, so that $U_{\mu}^{*}(h) \leqslant(h / \log (1+c)+1) \delta$.

\section{Concentration of maxima and minima in $L^{\alpha}$-norm}

\section{Proof of Corollary 1.2.}

Sufficiency. Assume that (1b) is true. Then, for the sequence $a_{n}=\mathrm{E} M_{n}$, or $a_{n}=m_{p}\left(M_{n}\right)$ (as stated at the end of Corollary 1.2), Chebyshev's inequality implies that $\sup _{n} P\left\{\mid M_{n}-\right.$ $\left.a_{n} \mid>h\right\} \rightarrow 0$ as $h \rightarrow+\infty$. Thus, property (1c) of Lemma 2.1 is fulfilled, so the function $U_{\mu}$ has finite modulus of continuity on the interval $[0,+\infty)$. Assumption $(2 b)$ is just $(1 b)$ for the sequence $\left(-\xi_{n}\right), n \geqslant 1$. Hence, again by Lemma 2.1 , the function $U_{\mu}$ has finite modulus of continuity on the interval $(-\infty, 0]$. As a result, $U_{\mu}$ has finite modulus on the whole real line. It now remains to make use of Theorem 1.1 .

Necessity. As before, let $M_{n}=\max \left\{\xi_{1}, \ldots, \xi_{n}\right\}$. Let $\zeta_{n}, n \geqslant 1$, be a sequence of independent random variables with common (logistic) distribution $v$, and let $Z_{n}=\max \left\{\zeta_{1}, \ldots, \zeta_{n}\right\}$. Since $U_{\mu}$ transforms $v$ into $\mu, M_{n}$ and $U_{\mu}\left(Z_{n}\right)$ are identically distributed. Therefore,

$$
\mathrm{E}\left|M_{n}-M_{n}^{\prime}\right|^{\alpha}=\mathrm{E}\left|U_{\mu}\left(Z_{n}\right)-U_{\mu}\left(Z_{n}^{\prime}\right)\right|^{\alpha},
$$

where $\left(M_{n}^{\prime}, Z_{n}^{\prime}\right)$ is an independent copy of $\left(M_{n}, Z_{n}\right)$. By Theorem 1.1, there exist constants $a, b \geqslant 0$ such that $\left|U_{\mu}(x)-U_{\mu}(y)\right| \leqslant a+b|x-y|$ whenever $x, y \in \mathbb{R}$. Thus, for all $\alpha \geqslant 1$, the left-hand side of (4.1) is bounded as $n \rightarrow \infty$, if the same is true for $Z_{n}$ instead of $M_{n}$. That is to say, we have reduced our attempt at a proof to the case $\mu=\nu$. So, one may assume 
that $\xi_{n}=\zeta_{n}$, and that $M_{n}=Z_{n}$, for all $n$. In this special case, $U_{\mu}$ is the identity function. Therefore, applying a remark following the statement of Theorem 1.1 to the functions $f_{n}(x)=\max _{1 \leqslant k \leqslant n} x_{k}$, there exist Lipschitz functions $U_{n}: \mathbb{R} \rightarrow \mathbb{R}$, with Lipschitz constants at most 1 , such that the random variables $M_{n}$ and $U_{n}\left(\xi_{1}\right)$ are identically distributed (of course, in this particular case, this is easily verified directly). Therefore, for all $n \geqslant 1$,

$$
\mathrm{E}\left|M_{n}-M_{n}^{\prime}\right|^{\alpha}=\mathrm{E}\left|U_{n}\left(\xi_{1}\right)-U_{n}\left(\xi_{1}^{\prime}\right)\right|^{\alpha} \leqslant \mathrm{E}\left|\xi_{1}-\xi_{1}^{\prime}\right|^{\alpha}=C_{\alpha},
$$

where $\xi_{1}^{\prime}$ is an independent copy of $\xi_{1}$. Now, by Hölder's inequality,

$$
\mathrm{E}\left|M_{n}-\mathrm{E} M_{n}\right|^{\alpha}=\mathrm{E}\left|\mathrm{E}^{\prime}\left(M_{n}(\omega)-M_{n}^{\prime}\left(\omega^{\prime}\right)\right)\right|^{\alpha} \leqslant \mathrm{E}\left|M_{n}-M_{n}^{\prime}\right|^{\alpha} \leqslant C_{\alpha},
$$

where $\mathrm{E}^{\prime}$ is taken with respect to the random variable $M_{n}^{\prime}$. This proves (1b). Property (2b) is proved in a similar way, taking into account that $v$ is symmetric about 0 . In order to prove the last statement on the quantiles, one can apply (1.3) to the cubes $\left\{x: x_{i} \leqslant\right.$ const., for all $i \leqslant n\}$. This gives

$$
\begin{gathered}
P\left\{M_{n}-m_{p}\left(M_{n}\right)>h^{*}\right\} \leqslant P\left\{\zeta_{1}-m_{p}\left(\zeta_{1}\right)>h\right\}, \\
P\left\{M_{n}-m_{p}\left(M_{n}\right)<-h^{*}\right\} \leqslant P\left\{\zeta_{1}-m_{p}\left(\zeta_{1}\right)<-h\right\},
\end{gathered}
$$

for all $p \in(0,1), h>0$. Since $h^{*} \leqslant a+b h$, these inequalities immediately imply that

$$
\sup _{n} \mathrm{E}\left|M_{n}-m_{p}\left(M_{n}\right)\right|^{\alpha}<+\infty \text {. }
$$

Corollary 1.2 follows.

One may wonder how to express (1b), the concentration property of maxima in $L^{\alpha}$-norm, separately from (2b). Using Corollary 1.2, one can derive the following description.

Corollary 4.1. Let $\mathrm{E}\left|\xi_{1}\right|^{\alpha}<+\infty, \alpha \geqslant 1$. The following are equivalent:

(1) $\sup _{n} \mathrm{E}\left|M_{n}-\mathrm{E} M_{n}\right|^{\alpha}<+\infty$.

(2) There exist $a, b \geqslant 0$ such that, for all $\frac{1}{2} \leqslant p \leqslant q<1$,

$$
F_{\mu}^{-1}(q)-F_{\mu}^{-1}(p) \leqslant a+b \log \frac{1-p}{1-q} .
$$

Corollary 4.1 (generalizing a statement of the authors in the case $\alpha=1$ ) and its elegant proof were kindly indicated to us by a referee. As also mentioned to us, these arguments also apply to more general norms.

Recall that $F_{\mu}^{-1}(p)$ is the minimal quantile of order $p$ of $F_{\mu}$. Thus, condition (4.2) expresses the fact that the function $U_{\mu}(x)=F_{\mu}^{-1}(1 /(1+\exp (-x)))$ sending $v$ into $\mu$ has a finite modulus of continuity on the interval $[0,+\infty)$. This implies that $\mathrm{E}\left(\xi_{1}^{+}\right)^{\alpha}<+\infty$, and moreover that $\operatorname{Eexp}\left(\varepsilon \xi_{1}\right)<+\infty$, for some $\varepsilon>0$ (as usual, $x^{+}=\max \{x, 0\}$ ). However, it says nothing about the behaviour of $F_{\mu}$ at $-\infty$.

Proof of Corollary 4.1. When $\xi_{i} \geqslant 0$, the proof is immediate since then $0 \leqslant \min \left\{\xi_{1}\right.$, $\left.\ldots, \xi_{n}\right\} \leqslant \xi_{1}$, which implies (2b). In general, one can observe that 


$$
M_{n}^{+}-\left|\xi_{1}\right| \leqslant M_{n} \leqslant M_{n}^{+}
$$

so that

$$
\left|M_{n}-\mathrm{E} M_{n}\right| \leqslant\left|M_{n}^{+}-\mathrm{E} M_{n}^{+}\right|+\left|\xi_{1}\right|+\mathrm{E}\left|\xi_{1}\right| .
$$

Therefore, $\sup _{n} \mathrm{E}\left|M_{n}-\mathrm{E} M_{n}\right|^{\alpha}<+\infty$ if and only if $\sup _{n} \mathrm{E}\left|M_{n}^{+}-\mathrm{E} M_{n}^{+}\right|^{\alpha}<+\infty$. On the other hand, $M_{n}^{+}=\max \left\{\zeta_{1}^{+}, \ldots, \xi_{n}^{+}\right\}$corresponds to non-negative random variables. Hence, by the previous step, the sequence $\mathrm{E}\left|M_{n}^{+}-\mathrm{E} M_{n}^{+}\right|^{\alpha}$ is bounded if and only if $U_{\mu}$ has finite modulus of continuity on $[0,+\infty)$. This finishes the proof.

In the case $\alpha=1$, condition (2) together with $\mathrm{E}\left|\xi_{1}\right|<+\infty$ can equivalently be written as one property:

$$
\int_{0}^{1} p^{n}(1-p) \mathrm{d} F_{\mu}^{-1}(p)=O\left(\frac{1}{n}\right), \quad \text { as } n \rightarrow \infty .
$$

In turn, this leads to another formulation.

Corollary 4.2. Let $\xi$ be a random variable with values in $(0,1)$ and with distribution function $F_{\xi}$. Then,

$$
\mathrm{E} \xi^{n}=O\left(\frac{1}{n}\right), \quad \text { as } n \rightarrow \infty,
$$

if and only if there exist $a, b \geqslant 0$ such that, for all $\frac{1}{2} \leqslant p \leqslant q<1$,

$$
\int_{p}^{q} \frac{1}{1-t} \mathrm{~d} F_{\xi}(t) \leqslant a+b \log \frac{1-p}{1-q} .
$$

\section{Concluding remarks}

Let $(X, \rho, \mu)$ be a metric space equipped with a Borel probability measure $\mu$. As in Section 1 , define the open $h$-neighbourhood of a set $A \subset X$ by

$$
A^{h}=\left\{x \in \mathbb{R}^{n}: \rho(x, a)<h \text { for some } a \in A\right\}, \quad h>0,
$$

and the associated ('integral') isoperimetric function

$$
R_{h}(p)=\inf _{\mu(A) \geqslant p} \mu\left(A^{h}\right), \quad 0<p<1,
$$

where the infimum is taken over all Borel sets of measure $\mu(A) \geqslant p$. With this notation, one can easily prove the following statement which contrasts with the $p-1$ alternative of Theorem 1.1, and deals with $n$ fixed.

Proposition 5.1. $R_{h}(p) \rightarrow 1$ as $h \rightarrow+\infty$ whenever $p \in(0,1)$.

This statement remains true if the metric is replaced by a pseudo-metric $\rho$ such that 
$\rho(x, y)<+\infty$, for almost all $(x, y)$ with respect to measure $\mu \otimes \mu$. In Section 1 , the concentration property $\inf _{n} R_{h}^{(n)}(p)>p$ could also have been expressed as $R_{h}(p)>p$, for the space $X=\mathbb{R}^{\infty}$ equipped with the pseudo-metric

$$
\rho_{\infty}(x, y)=\sup _{i \geqslant 1}\left|x_{i}-y_{i}\right|,
$$

and with the product measure $\mu^{\infty}$. In this case, $\rho(x, y)=+\infty$, for almost all $(x, y)$ with respect to $\mu^{\infty}$, whenever the measure $\mu$ does not have compact support on the real line.

\section{Acknowledgements}

The research of the first author was supported in part by the Alexander von Humboldt Foundation and the Russian Foundation for Fundamental Research, grant 96-01-00201. This author enjoyed the hospitality of the School of Mathematics, Georgia Institute of Technology, while part of the research was being carried out.

The research of the second author was supported in part by an NSF Postdoctoral Fellowship and by National Science Foundation grant no. DMS-9803239. This author enjoyed the hospitality of the Steklov Mathematical Institute (St Petersburg branch) and of the Department of Mathematics, University of Syktyvkar, while part of the research was being carried out.

We thank the referees for pointing out to us the work of de Haan and Ridder and for other important comments including Corollary 4.1, with $\alpha>1$, and its elegant proof.

\section{References}

Bobkov, S.G. (1996) Extremal properties of half-spaces for log-concave distributions. Ann. Probab., 24, 35-48.

Bobkov S.G. and Götze, F. (1999) Discrete isoperimetric and Poincaré-type inequalities. Probab. Theory Related Fields, 114, 245-277.

Bobkov, S.G. and Houdré, C. (1997) Some Connections between Sobolev-Type Inequalities and Isoperimetry, Mem. Amer. Math. Soc. 616. Providence, RI: American Mathematical Society.

Bobkov, S.G. and Ledoux, M. (1997) Poincaré inequalities and Talagrand's concentration phenomenon for the exponential distribution. Probab. Theory Related Fields, 107, 383-400.

de Haan, L. and Ridder, G. (1979) Stochastic compactness of sample extremes. Ann. Probab., 7, 290303.

Talagrand, M. (1991) A new isoperimetric inequality and the concentration of measure phenomenon. In J. Lindenstrauss and V.D. Milman (eds), Geometric Aspects of Functional Analysis, Lecture Notes in Math. 1469, pp. 94-124. Berlin: Springer-Verlag.

Talagrand, M. (1995) Concentration of measure and isoperimetric inequalities in product spaces. Inst. Hautes Études Sci. Publ. Math., 81, 73-205.

Received June 1996 and revised January 2000 